\title{
Bilayer Coherent and Quantum Hall Phases: Duality and Quantum Disorder
}

\section{Citation}

Demler, Eugene, Chetan Nayak, and S. Das Sarma. 2001. Bilayer Coherent and Quantum Hall Phases: Duality and Quantum Disorder. Physical Review Letters 86, no. 9: 1853-1856. doi:10.1103/physrevlett.86.1853.

\section{Published Version}

doi:10.1103/PhysRevLett.86.1853

\section{Permanent link}

http://nrs.harvard.edu/urn-3:HUL.InstRepos:34334613

\section{Terms of Use}

This article was downloaded from Harvard University's DASH repository, and is made available under the terms and conditions applicable to Other Posted Material, as set forth at http:// nrs.harvard.edu/urn-3:HUL.InstRepos:dash.current.terms-of-use\#LAA

\section{Share Your Story}

The Harvard community has made this article openly available.

Please share how this access benefits you. Submit a story.

\section{Accessibility}




\title{
Bilayer Coherent and Quantum Hall Phases: Duality and Quantum Disorder
}

\author{
Eugene Demler, ${ }^{1}$ Chetan Nayak, ${ }^{2}$ and S. Das Sarma ${ }^{3}$ \\ ${ }^{1}$ Physics Department, Harvard University, Cambridge, Massachusetts 02138 \\ ${ }^{2}$ Physics Department, University of California at Los Angeles, Los Angeles, California 90095 \\ ${ }^{3}$ Department of Physics, University of Maryland, College Park, Maryland 20742
}

(Received 9 August 2000)

\begin{abstract}
We consider a fully spin-polarized quantum Hall system with no interlayer tunneling at total filling factor $\nu=1 / k$ (where $k$ is an odd integer) using the Chern-Simons-Ginzburg-Landau theory. Exploiting particle-vortex duality and the concept of quantum disordering, we find a large number of possible compressible and incompressible ground states, some of which may have relevance to recent experiments of Spielman et al. [Phys. Rev. Lett. 84, 5808 (2000)]. We find interlayer coherent compressible states without Hall quantization and interlayer incoherent incompressible states with Hall quantization in addition to the usual $(k, k, k)$ Halperin states, which are both interlayer coherent and incompressible.
\end{abstract}

DOI: 10.1103/PhysRevLett.86.1853

Bilayer quantum Hall systems have been a subject of great experimental and theoretical interest $[1-10]$ for more than a decade. In particular, spin-polarized bilayer quantum Hall systems with little or no interlayer tunneling $\left(\Delta_{\mathrm{SAS}} \approx 0\right)$ at the total Landau-level filling factor $\nu=$ 1 have been studied intensively because the layer index serves as a pseudospin index with U(1) symmetry, leading to the possibility of many interesting quantum phases and quantum phase transitions associated with it. Among the many exotic possibilities discussed in the literature in this context are long-range pseudospin order (the so-called spontaneous interlayer phase coherence) associated with the spontaneous breaking of the pseudospin U(1) symmetry, a linearly dispersing collective Goldstone mode, bilayer pseudospin superfluidity, the associated Josephson effect, topological defects (merons, Skyrmions, and vortices), and a Kosterlitz-Thouless phase transition at finite temperature. The subject is of considerable current interest as a result of the recent appearance [11] of an experimental paper reporting the possible observation in a bilayer tunneling measurement of the predicted Goldstone mode at $\nu=1$ associated with pseudospin U(1) symmetry breaking. There have been several recent preprints [12] providing possible theoretical explanations of the data presented in [11].

In this Letter, we consider a somewhat more general situation (still with $\Delta_{\mathrm{SAS}}=0$, although a generalization to $\Delta_{\mathrm{SAS}} \neq 0$ is straightforward) with total filling factor $\nu=1 / k$ (with $k$ an odd integer) from a new theoretical perspective hitherto not considered in the literature. We extend the Chern-Simons-Landau-Ginzburg (CSLG) theory for the Halperin $(k, k, k)$ state [6,13] to describe the possibility of quantum disordering of the charge and pseudospin degrees of freedom. Our quantum disordering procedure relies on the $\mathrm{U}(1)$ particle-vortex duality in $(2+1)$ dimensions and leads to a large class of incompressible and compressible bilayer states at $\nu=1 / k$; some are interlayer coherent, and others are not. One of our important conclusions is that even for $\Delta_{\mathrm{SAS}}=0$ the existence
PACS numbers: 73.43.-f, 71.10.Pm, 73.21.-b

of interlayer coherence is neither necessary nor sufficient for the existence of an incompressible bilayer $\nu=1 / k$ quantum Hall state. It is possible to have Hall quantization at $\nu=1 / k$ without having interlayer coherence and vice versa. We believe that this important result of ours may have implications for the experimental observations in Ref. [11], where the putative Goldstone mode associated with interlayer coherence has presumably been observed in a state which is either compressible or is very weakly incompressible. We therefore raise the interesting (but by no means definitive) possibility that the bilayer system in Ref. [11] is not the Halperin $(1,1,1)$ ground state, as has been universally assumed, in which interlayer coherence and incompressibility occur together, but is one of the new quantum disordered interlayer coherent and compressible states (e.g., the Hall insulator [14] state we find below) predicted in this paper. The experimental data presented in Ref. [11] provide some circumstantial evidence in support of such a tentative claim: (1) the $\nu=1$ zero-bias tunneling conductance peak reported in [11] is much broader than the weak $(>1 \mathrm{k} \Omega)$ and shallow $\rho_{x x}$ minimum seen in the data ( $\rho_{x y}$ quantization has not been observed) even at the very low temperature of $40 \mathrm{mK}$; $(2)$ the zero-bias tunneling peak width exists [11] over $\Delta \nu \approx 0.7$ and, in fact, contains even the $\rho_{x x}$ maximum within it, raising some doubts about the standard interpretation of the state as the $(1,1,1)$ state, which is manifestly an incompressible state; (3) $\rho_{x x}$ for $\nu<1$ in Ref. [11] shows striking insulating behavior as it increases extremely sharply with decreasing $\nu$, indicating that it may be a Hall insulator for these $\nu$. We point out that all the quantum disordered compressible states found by us break translational invariance. In the clean limit, they are Wigner crystal/charge-density-wave $(\mathrm{CDW}) /$ striped phase-type states in each layer. In the limit that disorder plays a large role (a more likely scenario for Ref. [11] where the carrier density is very low and consequently disorder effects may be important), they are better described as disorder-induced localized phases. We believe that some of the bilayer striped (compressible) 
interlayer coherent phases recently discussed in the literature [15] within a microscopic Hartree-Fock calculation may actually belong to the class of translational invariancebroken compressible coherent quantum disordered phases we find below.

Our construction has precedents in the high- $T_{c}$ superconductivity literature [16], where it has been used to describe spin-charge separation. Here we implement pseudospin-charge separation to disorder the pseudospin and charge currents separately. In the context of superconductivity (or superfluidity), it is clear that magnetism and superconductivity (or superfluidity) are distinct effects. The triplet order parameter of the $A$ phase of ${ }^{3} \mathrm{He}$ breaks both spin and particle number symmetries, but these symmetries may be restored separately; restoring the latter alone would lead to a magnetic state of solid helium. Our construction can be understood in a similar vein, with the interlayer coherent $(1,1,1)$ quantum Hall state replacing triplet superconductivity. Our work is also related to the hierarchy construction of Lee and Kane [13], but these authors considered only the condensation of charged vortices and Skyrmions, which does not lead to our cornucopia of states with the same total $\sigma_{x y}$.

We begin our discussion of bilayer quantum Hall systems at $\nu=1 / k$ with a CSLG theory $[6,13,17]$ that describes incompressible interlayer coherent states with the Halperin $(k, k, k)$ wave function. We neglect real spin, assuming complete spin polarization, but allow unequal layer populations, i.e., the so-called unbalanced case, $\rho_{1} \neq$ $\rho_{2}$, where $\rho_{i}$ is the electron density in layer $i$. The imaginary-time action for this theory is $(\hbar=c=e=1)$

$$
\begin{aligned}
\mathcal{L}= & \sum_{i=1,2}\left\{\Psi_{i}^{\dagger}\left(\partial_{0}-i a_{0}-i A_{0}^{(i)}\right) \Psi_{i}+\frac{1}{2 m}\left|\left[\vec{\partial}-i \vec{a}-i \vec{A}^{(i)}\right] \Psi_{i}\right|^{2}+\frac{U}{2}\left(\Psi_{i}^{\dagger} \Psi_{i}-\rho_{i}\right)^{2}\right\} \\
& +V\left(\Psi_{1}^{\dagger} \Psi_{1}-\rho_{1}\right)\left(\Psi_{2}^{\dagger} \Psi_{2}-\rho_{2}\right)-\frac{i}{4 \pi k} \epsilon_{\mu \nu \lambda} a_{\mu} \partial_{\nu} a_{\lambda},
\end{aligned}
$$

where the $\Psi_{i}$ 's are the bosonic fields which describe electrons in the two layers and the final term in (1) is the Chern-Simons term, $\mathcal{L}_{\mathrm{CS}}(a)$, which enforces the fermionic statistics of the electrons. The gauge fields $A_{\mu}^{(1,2)}$ couple to electrons in layers 1 and 2 , respectively. By choosing $\rho_{1}$ and $\rho_{2}$ to be arbitrary we allow for charge imbalance between the layers. For simplicity, we replace the long-range Coulomb interaction by short-range interactions. Intralayer interactions are not equal to interlayer interactions, $U \neq V$, so the model has only $\mathrm{U}(1)$ pseudospin symmetry, rather than the full SU(2) which would be obtained if $U=V$.

We take $\Psi_{i}=\sqrt{\rho_{i}} e^{i \theta_{i}}$ and introduce $\theta=$ $\left(\theta_{1}+\theta_{2}\right) / 2, \quad \phi=\left(\theta_{1}-\theta_{2}\right) / 2$. Pseudospin $\mathrm{U}(1)$ is the symmetry $\phi \rightarrow \phi+$ const. When $\phi$ spontaneously chooses a direction, this symmetry is broken and the oscillations of $\phi$ describe the Goldstone mode associated with this broken symmetry. Similarly, we define $A_{\mu}^{C}=$ $\left(A_{\mu}^{(1)}+A_{\mu}^{(2)}\right) / 2$ and $A_{\mu}^{I}=\left(A_{\mu}^{(1)}-A_{\mu}^{(2)}\right) / 2 . \quad A^{C}$ couples to the total charge of the system, while $A^{I}$ couples to the charge difference between the two layers, i.e., the pseudospin. Vortices in $\theta_{1}$ or $\theta_{2}$ are called merons [6]. They come in four varieties since they can be either vortices or antivortices in $\theta_{1}$ or $\theta_{2}$, and they have charge $\pm \rho_{1,2} /\left[\left(\rho_{1}+\rho_{2}\right) k\right]$. Vortices in $\theta_{1}$ and $\theta_{2}$ can be combined to form a vortex in $\theta$. In pseudospin language, they are Skyrmions [6], carrying charge $\pm 1 / k$. A vortex in $\theta_{1}$ can be combined with an antivortex in $\theta_{2}$ to form a vortex in $\phi$ of charge $\left(\rho_{1}-\rho_{2}\right) /\left[\left(\rho_{1}+\rho_{2}\right) k\right]$.

If we integrate out the amplitude fluctuations in (1) and set $\left(\rho_{1}+\rho_{2}\right) / m=1, K_{3}=\left(\rho_{1}-\rho_{2}\right) /\left(\rho_{1}+\rho_{2}\right)$, we obtain

$$
\begin{aligned}
\mathcal{L}= & \frac{1}{2 v_{c}^{2}}\left(\partial_{0} \theta-a_{0}-A_{0}^{C}\right)^{2}+\frac{1}{2 v_{s}^{2}}\left(\partial_{0} \phi-A_{0}^{I}\right)^{2}+\frac{1}{2}\left(\vec{\partial} \theta-\vec{a}-\vec{A}^{C}\right)^{2}+\frac{1}{2}\left(\vec{\partial} \phi-\vec{A}^{I}\right)^{2} \\
& +K_{3}\left(\vec{\partial} \theta-\vec{a}-\vec{A}^{C}\right) \cdot\left(\vec{\partial} \phi-\vec{A}^{I}\right)-\mathcal{L}_{\mathrm{CS}}(a),
\end{aligned}
$$

where $v_{c, s}$ are the velocities of the charge and pseudospin collective modes.

Using the standard U(1) particle-vortex duality [18] for both $\theta$ and $\phi$ and their vortex excitations, we rewrite (2) as

$$
\begin{aligned}
\tilde{\mathcal{L}}_{D}= & \frac{\rho_{C}}{2}\left|\left(\partial_{\mu}-i b_{\mu}^{C}\right) \Phi_{C}\right|^{2}+\frac{\rho_{I}}{2}\left|\left(\partial_{\mu}-i b_{\mu}^{I}\right) \Phi_{I}\right|^{2}+\frac{\kappa_{0}}{4}\left(F_{\alpha \beta}^{C}\right)^{2}-\pi k i \epsilon_{\mu \nu \lambda} b_{\mu}^{C} \partial_{\nu} b_{\lambda}^{C}-i b_{\mu}^{C} \epsilon_{\mu \nu \lambda} \partial_{\nu} A_{\lambda}^{C} \\
& +\frac{\kappa_{0}}{4}\left(F_{\alpha \beta}^{I}\right)^{2}-i b_{\mu}^{I} \epsilon_{\mu \nu \lambda} \partial_{\nu} A_{\lambda}^{I}-\kappa_{3} F_{0 \alpha}^{C} F_{0 \alpha}^{I},
\end{aligned}
$$

where $\kappa_{0}=1 /\left(1-K_{3}^{2}\right), \kappa_{3}=K_{3} /\left(1-K_{3}^{2}\right)$, and $F_{\alpha \beta}^{C, I}$ is the field strength associated with $b_{\mu}^{C, I}$. (To avoid clutter, we set $v_{c, s}=1$; they may be restored by dividing all temporal derivatives by the appropriate velocity.) The fields in (3) are related to those of (2) according to 


$$
\begin{gathered}
\epsilon_{\mu \nu \lambda} \partial_{\nu} b_{\lambda}^{C} \equiv \partial_{\mu} \theta-a_{\mu}-A_{\mu}^{C}+\left(1-\delta_{\mu 0}\right) K_{3}\left(\partial_{\mu} \phi-A_{\mu}^{I}\right), \\
\epsilon_{\mu \nu \lambda} \partial_{\nu} b_{\lambda}^{I} \equiv \partial_{\mu} \phi-A_{\mu}^{I}+\left(1-\delta_{\mu 0}\right) K_{3}\left(\partial_{\mu} \theta-a_{\mu}-A_{\mu}^{C}\right), \\
\rho_{C, I} \operatorname{Im}\left[\Phi_{C, I}^{*}\left(\partial_{\mu}-i b_{\mu}^{C, I}\right) \Phi_{C, I}\right] \equiv \epsilon_{\mu \nu \lambda} \partial_{\nu} \partial_{\lambda}\{\theta, \varphi\} .
\end{gathered}
$$

The right-hand sides of the first two equations are, respectively, the conserved charge and pseudospin currents of (2). $\Phi_{C, I}$ create vortices in $\theta$ or $\phi$, respectively. We have implicitly assumed that vortices in $\theta_{1,2}$ (i.e., merons) are higher in energy and can, therefore, be neglected. As in the high- $T_{c}$ case [16], this assumption leads to chargepseudospin separation and a particular form of topological order [19]. We want to consider the more general situation in which the lowest energy excitation is a composite formed by $n$ vortices in $\theta$ and $m$ vortices in $\phi$, $\Phi_{n, m} \sim \Phi_{C}^{n} \Phi_{I}^{m}$, where $n, m$ are integers. In this case, we may write the effective action as

$$
\begin{aligned}
\tilde{\mathcal{L}}_{D}= & \frac{1}{2} \rho_{n, m}\left|\left(\partial_{\mu}-i n b_{\mu}^{C}-i m b_{\mu}^{I}\right) \Phi_{n, m}\right|^{2} \\
& +\frac{\kappa_{0}}{4}\left(F_{\alpha \beta}^{C}\right)^{2}-\pi k i \epsilon_{\mu \nu \lambda} b_{\mu}^{C} \partial_{\nu} b_{\lambda}^{C}-i b_{\mu}^{C} \epsilon_{\mu \nu \lambda} \partial_{\nu} A_{\lambda}^{C} \\
& +\frac{\kappa_{0}}{4}\left(F_{\alpha \beta}^{I}\right)^{2}-i b_{\mu}^{I} \epsilon_{\mu \nu \lambda} \partial_{\nu} A_{\lambda}^{I}-\kappa_{3} F_{0 \alpha}^{C} F_{0 \alpha}^{I}
\end{aligned}
$$

When there is no vortex condensate, $\left\langle\Phi_{n, m}\right\rangle=0$ for all $n, m$, we may drop the vortex part of the action and obtain the response functions from the remaining (quadratic) terms in $b_{\mu}^{C}, b_{\mu}^{I}$ :

$$
\begin{array}{cc}
\sigma_{x x}^{C C}=0, & \sigma_{x y}^{C C}=\frac{1}{2 \pi k}, \\
\sigma_{x x}^{I C}=0, & \sigma_{x y}^{I C}=\frac{\kappa_{3}}{\kappa_{0}} \frac{1}{2 \pi k}, \\
\sigma_{x x}^{I I}=\frac{1}{\kappa_{0}} \frac{i}{\omega}, & \sigma_{x y}^{I I}=\left(\frac{\kappa_{3}}{\kappa_{0}}\right)^{2} \frac{1}{2 \pi k} .
\end{array}
$$

Equations (6) tell us that we have an incompressible quantum liquid with quantized Hall conductance. The first of Eqs. (8) describes an interlayer superfluid with a singularity at zero frequency due to the Goldstone mode associated with broken pseudospin symmetry. It is remarkable that our simple analysis allows us to calculate the weight of this mode as a function of charge imbalance between the layers

$$
I\left(\rho_{1}-\rho_{2}\right)=\frac{1}{\kappa_{0}}=I_{0}\left[1-\left(\frac{\rho_{1}-\rho_{2}}{\rho_{1}+\rho_{2}}\right)^{2}\right] .
$$

This is a simple, experimentally verifiable prediction of our theory.

Various quantum disordered phases of the $(k, k, k)$ state can also be described using (5). They result when $\Phi_{n, m}$ condenses for some $n, m$. When this occurs, the gauge field $n b_{\mu}^{C}+m b_{\mu}^{I}$ acquires a gap. After integrating it out, we obtain the response functions of the corresponding quantum disordered phases which we discuss below. A mean-field Landau-Ginzburg analysis is justified because the fluctuations of $b_{\mu}^{C}$ are suppressed either by incompressibility or the Anderson-Higgs effect associated with the condensation of $\Phi_{n, 0}$. The fluctuations of $b_{\mu}^{I}$ are either gapped (interlayer incoherent states) or are simply those of a Goldstone mode (interlayer coherent states).

When $m=0$ and $n \neq 0$ we find that we destroy the quantum Hall effect without destroying interlayer coherence, $\sigma_{x x}^{C C}=\sigma_{x y}^{C C}=0$ and $\sigma_{x x}^{I I}=\frac{1}{\kappa_{0}} \frac{i}{\omega}$. So, relation (9) will be satisfied even when the state becomes compressible. As we mentioned in our introductory comments, this state may be relevant to the experiment of [11]. Since the flux of $b_{\lambda}^{C}$ is fixed by $\epsilon_{0 \nu \lambda} \partial_{\nu} b_{\lambda}^{C}=\rho_{1}+\rho_{2}$, it must penetrate the $\Phi_{n, 0}$ condensate. As a result, translational symmetry must be broken, either spontaneously by Wigner crystallization or manifestly by disorder. In the perfectly clean limit, the flux of $b_{\lambda}^{C}$ enters the $\Phi_{n, 0}$ condensate in an analog of the Abrikosov flux lattice. From (5), we see that flux tubes in $\Phi_{n, 0}$ carry flux $2 \pi / n$ and, hence, charge $1 / n$, equally distributed between the two layers. Hence, this is a Wigner crystal of charge $1 / n$ quasiparticles which is coherent between the two layers. In the strong disorder limit, this can be viewed as a localized phase of charge $1 / n$ quasiparticles.

When $m \neq 0$, we find that interlayer coherence is destroyed but the quantum Hall effect is not

$$
\begin{array}{cc}
\sigma_{x x}^{C C}=0, & \sigma_{x y}^{C C}=\frac{1}{2 \pi k}, \\
\sigma_{x x}^{I C}=0, & \sigma_{x y}^{I C}=\frac{n}{m} \frac{1}{2 \pi k}, \\
\sigma_{x x}^{I I}=0, & \sigma_{x y}^{I I}=\left(\frac{n}{m}\right)^{2} \frac{1}{2 \pi k} .
\end{array}
$$

At small frequencies, $\sigma_{x x}^{I I} \propto i \omega$ so this phase corresponds to an interlayer insulator, rather than a superfluid. We notice the following remarkable property shared by interlayer coherent and incoherent states: $\sigma_{x y}^{C C} \sigma_{x y}^{I I}=\left(\sigma_{x y}^{I C}\right)^{2}$. When interlayer coherence is destroyed, the pseudospin Hall conductances are quantized. Since a vortex corresponding to $\Phi_{0,1}, \Phi_{0,2}, \ldots, \Phi_{0, m-1}$, or no vortex at all can be threaded along either of the noncontractible loops of the torus, there is an $\mathrm{m}^{2}$-fold ground state degeneracy in the pseudospin sector which, when combined with the $k$-fold degeneracy of the charge sector, gives a total ground state degeneracy of $\mathrm{km}^{2}$ on the torus. For general $n, m$, these states break translational invariance. However, in the case $n=0$, $m \neq 0, \epsilon_{0 \nu \lambda} \partial_{\nu} b_{\lambda}^{I}=\rho_{1}-\rho_{2}$, so translational symmetry will be broken only if the layers are unbalanced, in which case the charge difference between the layers will be modulated.

We note that there is a straightforward generalization akin to the hierarchy construction which involves the introduction of an additional Chern-Simons field, $\beta_{\mu}$, which attaches $2 l$ flux tubes to $\Phi_{n, m}$. Our earlier construction 
was simply the $l=0$ case. The $l \neq 0$ modification suggests a new set of states with the same conductances as in (10) and (11), but with $\sigma_{x y}^{I I}(12)$ replaced by $\sigma_{x y}^{I I}=$ $(1 / 2 \pi k)\left(n^{2}-2 l k\right) / m^{2}$. It may be shown, using standard arguments of CSLG theory [20], that these states have a ground state degeneracy of $\mathrm{km}^{2}$ on the torus; this is independent of $l$ and is the same as in the $l=0$ case. When $m=0$, we obtain an interlayer coherent state with $\sigma_{x y}^{C C}=2 l / 2 \pi(2 l k \pm 1)$.

A physical interpretation for many of these states may be given by considering a quantum Hall state obtained by condensing composites which consist of $p$ electrons in layer 1 and $q$ electrons in layer 2. We assume that the composite is tightly bound so that we can ignore its internal structure. Let $\Psi_{c}$ be the operator that creates the auxiliary boson defined by statistical transmutation of such a composite. The CSLG theory for $\Psi_{c}$ takes the form

$$
\begin{aligned}
\mathcal{L}_{c}= & \Psi_{c}^{\dagger}\left(\partial_{0}-a_{0}-p A_{0}^{(1)}-q A_{0}^{(2)}\right) \Psi_{c} \\
& +\frac{1}{2 m}\left|\left(\vec{\partial}-i \vec{a}-i p \vec{A}^{(1)}-i q \vec{A}^{(2)}\right) \Psi_{c}\right|^{2} \\
& -\frac{i}{4 \pi k_{c}} \epsilon_{\mu \nu \lambda} a_{\mu} \partial_{\nu} a_{\lambda} .
\end{aligned}
$$

If we take $k_{c}=k(p+q)^{2}$, then we have a quantum Hall state with $\sigma_{x y}^{C C}=1 /(2 \pi k)$. To ensure that the composite has the correct statistics, we must have $k$ odd. For the particular case of quasiparticle pairs, this relation has been discussed by Halperin in the context of Laughlin's wave functions [21]. Using (13), we find the response functions of the quantum Hall state of composite objects $\sigma_{x y}^{C C}=\frac{1}{2 \pi k}, \sigma_{x y}^{I C}=\frac{1}{2 \pi k} \frac{(p-q)}{(p+q)}$, and $\sigma_{x y}^{I I}=\frac{1}{2 \pi k} \frac{(p-q)^{2}}{(p+q)^{2}}$. By proceeding to the dual Chern-Simons theory, we deduce a ground state degeneracy of $k(p+q)^{2}$ on the torus. Hence, we find precisely the same conductance tensor and ground state degeneracy on the torus which we found earlier, with $p+q=m$ and $p-q=n$.

Let us consider two special cases. For $p=1$ and $q=0$, so that quantum liquid is in one layer only, we have $m=1$, and $n=1$, so it may be described by the condensation of a composite formed by one vortex in $\theta$ and one vortex in $\varphi$, i.e., a double vortex in $\theta_{1}$. When $p=1$ and $q=1$ we have the paired state suggested in [8]. It is described by $m=2$ and $n=0$, i.e., by the condensation of double vortices in $\varphi$. In this case, we see from (5) that when the layers are unbalanced, the charge difference enters in a Wigner crystal of isospin $1 / 2$ quasiparticles (charge difference between the layers $e / 2$ ).

To summarize, we have used U(1) particle-vortex duality to extend a CSLG theory for $\nu=1 / k$ (with $k$ an odd integer) for bilayer quantum Hall systems to discuss the states in which either Hall quantization or interlayer coherence ("pseudospin superfluidity") is individually destroyed. Our most important new conceptual results are the identification of the theoretical possibility that there may be $\nu=1$ (or $1 / k$ ) bilayer pseudospin coherent states which are compressible [unlike the usual $(1,1,1)$ state which is incompressible and interlayer coherent] and the observation that the experimental data presented in Ref. [11] are not manifestly inconsistent with the exciting prospect that such a pseudospin-coherent compressible state (most likely a disorder-driven Hall insulating phase) may actually be playing a role in Ref. [11].

It is a great pleasure to acknowledge discussions with B. Halperin, J.P. Eisenstein, and N. Read. C.N. and S.D. S. acknowledge the Aspen Center for Physics. E. D. is supported by the Harvard Society of Fellows. C. N. is supported by the National Science Foundation under Grant No. DMR-9983544 and the Alfred P. Sloan Foundation. S.D. S. is supported by the Office of Naval Research.

[1] See, for example, the articles by J.P. Eisenstein, S. M. Girvin, and A.H. MacDonald, in Perspectives in Quantum Hall Effects, edited by S. Das Sarma and A. Pinczuk (Wiley, New York, 1997), and references therein.

[2] H. A. Fertig, Phys. Rev. B 40, 1087 (1989).

[3] X. G. Wen and A. Zee, Phys. Rev. Lett. 69, 1811 (1992); Phys. Rev. B 47, 2265 (1993).

[4] Z. F. Ezawa and A. Iwazaki, Int. J. Mod. Phys. B 6, 3205 (1992).

[5] S. He et al., Phys. Rev. B 47, 4394 (1993); Y. W. Suen et al., Phys. Rev. Lett. 68, 1379 (1992); J. P. Eisenstein et al., Phys. Rev. Lett. 68, 1383 (1992).

[6] K. Moon et al., Phys. Rev. B 51, 5138 (1995); K. Yang et al., Phys. Rev. B 54, 11644 (1996).

[7] S. Q. Murphy et al., Phys. Rev. B 52, 14825 (1995).

[8] N. E. Bonesteel et al., Phys. Rev. Lett. 77, 3009 (1996).

[9] S. Das Sarma et al., Phys. Rev. Lett. 79, 917 (1997); Phys. Rev. B 58, 4672 (1998).

[10] A. Stern et al., Phys. Rev. Lett. 84, 139 (2000).

[11] I. B. Spielman et al., Phys. Rev. Lett. 84, 5808 (2000).

[12] J. Schliemann, preceding Letter, Phys. Rev. Lett. 86, 1849 (2001); A. Stern et al., cond-mat/0006457; L. Balents and L. Radzihovsky, Phys. Rev. Lett. 86, 1825 (2001); M. M. Fogler and F. Wilczek, Phys. Rev. Lett. 86, 1833 (2001).

[13] D.-H. Lee and C. L. Kane, Phys. Rev. Lett. 64, 1313 (1990).

[14] S. Kivelson, D.-H. Lee, and S.-C. Zhang, Phys. Rev. B 46, 2223 (1992).

[15] L. Brey and H. A. Fertig, cond-mat/0002218.

[16] L. Balents, M. P. A. Fisher, and C. Nayak, Phys. Rev. B 60, 1654 (1999); 61, 6307 (2000); E. Demler, C. Nayak, H.-Y. Kee, Y. B. Kim, and T. Senthil (to be published).

[17] S. C. Zhang et al., Phys. Rev. Lett. 62, 82 (1989); N. Read, Phys. Rev. Lett. 62, 86 (1989).

[18] M.P. A. Fisher and D.-H. Lee, Phys. Rev. B 39, 2756 (1989), and references therein.

[19] This can be made more explicit by introducing operators $\Phi_{1,2}$ which create vortices in $\theta_{1,2}$, so that $\Phi_{C}=\Phi_{1} \Phi_{2}$ while $\Phi_{I}=\Phi_{1} \Phi_{2}^{*}$. Then, our form of topological order (charge-pseudospin separation) is equivalent to the preservation of the $Z_{2}$ symmetry $\Phi_{1,2} \rightarrow-\Phi_{1,2}$.

[20] N. Read, Phys. Rev. Lett. 65, 1502 (1990); X. G. Wen and A. Zee, Phys. Rev. B 46, 2290 (1992).

[21] B. I. Halperin, Helv. Phys. Acta 56, 75 (1983). 